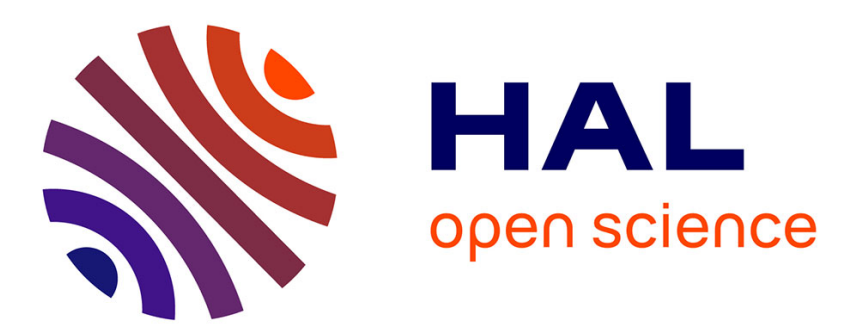

\title{
Dynamic fracture: an example of convergence towards a discontinuous quasi-static solution
}

Pierre-Emmanuel Dumouchel, Jean-Jacques Marigo, M. Charlotte

\section{To cite this version:}

Pierre-Emmanuel Dumouchel, Jean-Jacques Marigo, M. Charlotte. Dynamic fracture: an example of convergence towards a discontinuous quasi-static solution. Continuum Mechanics and Thermodynamics, 2008, 20, pp.1-19. 10.1007/s00161-008-0071-3 . hal-00551075

\section{HAL Id: hal-00551075 \\ https://hal-polytechnique.archives-ouvertes.fr/hal-00551075}

Submitted on 3 Jan 2011

HAL is a multi-disciplinary open access archive for the deposit and dissemination of scientific research documents, whether they are published or not. The documents may come from teaching and research institutions in France or abroad, or from public or private research centers.
L'archive ouverte pluridisciplinaire HAL, est destinée au dépôt et à la diffusion de documents scientifiques de niveau recherche, publiés ou non, émanant des établissements d'enseignement et de recherche français ou étrangers, des laboratoires publics ou privés. 


\title{
Dynamic fracture: an example of convergence towards a discontinuous quasi-static solution
}

\author{
P.-E. Dumouchel ${ }^{\text {a }}$ J.-J. Marigo ${ }^{\text {a, } *}$ \\ ${ }^{a}$ Institut Jean le Rond d'Alembert, Université Paris VI, 75005 Paris \\ M. Charlotte ${ }^{\mathrm{b}}$ \\ ${ }^{\mathrm{b}}$ Laboratoire de Mécanique des Solides, Ecole Polytechnique, 91128 Palaiseau
}

\begin{abstract}
Considering a one-dimensional problem of debonding of a thin film in the context of Griffith's theory, we show that the dynamical solution converges, when the speed of loading goes down to 0, to a quasi-static solution including an unstable phase of propagation. In particular, the jump of the debonding induced by this instability is governed by a principle of conservation of the total quasi-static energy, the kinetic energy being negligible.
\end{abstract}

\section{Introduction}

Griffith's theory of fracture mechanics is universally used to treat the propagation of cracks in brittle elastic bodies. When the load varies slowly with time, the quasi-static approach is preferred to the dynamic approach for its simplicity. But it turns out that, even when the crack path is known in advance, Griffith's law is unable to give the evolution of the crack if the total (quasi-static) energy is not a convex function of the crack length. Indeed, in this case, an unstable phase of propagation takes place and the quasi-static Griffith law, which does not allow discontinuous crack evolutions, cannot be applied any more. It is generally claimed that in a such situation the quasistatic framework must be abandoned because the crack propagation becomes dynamic and that the kinetic energy plays an important role. The goal of the paper is to show that, in a one-dimensional particular context, that claim is not true and that the unstable phase of propagation can also be treated

* Corresponding author. E-mail: marigo@lmm.jussieu.fr 
by considering only quasi-static energies. But the price to pay is to replace the Griffith law of propagation based on the concept of critical energy release rate by that of conservation of the total quasi-static energy, at least during the phases of unstable propagation. This result is proved by solving the full dynamical problem and by passing to the limit when the loading speed goes down to zero. To our best knowlegde, a similar argument has been used only by Berry $[1,2]$ in his approximate analysis of dynamic fracture (in 2D), which refered to a quasi-static $G$ for evaluating the kinetic energy and the fracture energy at crack arrest in an homogeneous medium. Consequently, that gives a theoretical basis to the variational approach suggested by [3], [4] and [5] where the crack evolution is governed by two criteria: a stability criterion and an energy balance. Indeed, the variational formulation can be applied even if the total quasi-static energy is a non convex function of the crack length, the jumps of the crack are governed by that energy balance whereas the two criteria are equivalent to Griffith's law when the crack length evolves smoothly.

The paper is organized as follows. In Section 2, we first introduce the onedimensional problem which corresponds to a simplified version of the peeling test. Then, we set the dynamic problem and introduce the two cases of toughness repartition that are considered in the sequel, i.e. the so-called Case a and Case b. Follows a discussion where it is shown that we cannot solve the non convex Case b in a quasi-static context by using Griffith's law alone because the debonding evolution is necessarily discontinuous. As an alternative the energy conservation principle is proposed to determine the value of the jump. Section 3 is devoted to the dynamic analysis of each case. We first introduce the general structure of the dynamic response made of a sequence of shock waves which interact with the front of debonding. Then, we obtain the solutions in a closed form. Finally, we pass to the limit when the speed of loading goes down to 0 to prove the convergence to the quasi-static solutions in each case.

\section{Setting of the problem}

\subsection{The dynamical problem}

\subsubsection{Notation and main assumptions}

The problem deals with a simplified version of the peeling test, cf [6], [7], [8] and Figure 1. We consider a semi-infinite perfectly flexible and inextensible thin film which is initially perfectly bonded to a rigid substrate with normal $\mathbf{e}_{2}$. The end $x_{1}=0$ of the film is submitted both to a constant tension $-N \mathbf{e}_{1}, N>$ 


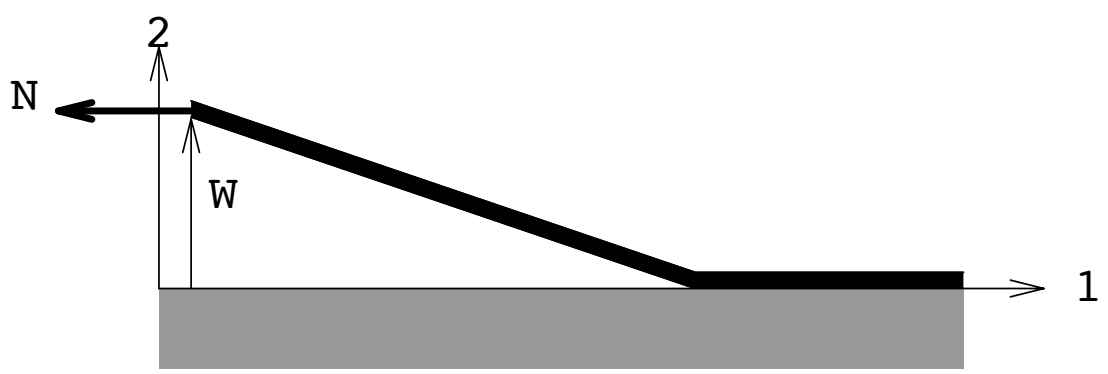

Fig. 1. Debonding of a thin inextensible film.

0 , and to an opening $W$ linearly increasing with time $t$ so that

$$
W=\epsilon c t, \quad c=\sqrt{\frac{N}{\rho}} .
$$

In (1), $\rho$ is the mass of a unit length of the film, $c$ is the velocity of the transversal waves propagating in the film and $\epsilon$ represents the dimensionless speed of loading. In the sequel, $\epsilon$ will be considered as a (small) parameter. Therefore, the dependence of the response on $\epsilon$ is made explicit by denoting the solution with $\epsilon$ as a superscript. The main goal of the paper is to determine the asymptotic behavior of the dynamical response when $\epsilon$ goes down to 0 . Moreover, in order to simplify the expressions we introduce dimensionless quantities. Specifically, if $L$ denotes a characteristic length of the film (which will correspond to the point where there is a change of toughness in the model developed below), we set

$$
x=\frac{x_{1}}{L}, \quad T=\epsilon \frac{c t}{L}
$$

and consider that the displacement field $\mathbf{u}^{\epsilon}$ of the film is a function of $(x, T)$ defined on the quadrant $\mathcal{Q}:=(0,+\infty)^{2}$,

$$
\mathbf{u}^{\epsilon}(x, T)=u^{\epsilon}(x, T) L \mathbf{e}_{\mathbf{1}}+w^{\epsilon}(x, T) L \mathbf{e}_{\mathbf{2}} .
$$

In (2), $T$ can be considered as a dimensionless rescaled time. Assuming that $w^{\epsilon}$ is small and using a linearized inextensibility condition, $u^{\epsilon}$ can be expressed in terms of $w^{\epsilon}$ as follows:

$$
u^{\epsilon}(x, T)=\frac{1}{2} \int_{x}^{\infty}\left(\frac{\partial w^{\epsilon}}{\partial x}\right)^{2}(s, T) d s .
$$

The debonded part of the film at time $t$ corresponds to the points $x$ such that $\sup _{S \leq T} w^{\epsilon}(x, S)>0$. To simplify the presentation, we assume that the debonding grows from $x=0$ at $T=0$ in such a way that the debonded part of the film at time $t$ corresponds to the interval $\left(0, \ell^{\epsilon}(T)\right)$. Thus $\ell^{\epsilon}(T) L$ 
represents the physical debonded length at time $t$. Therefore,

$$
u^{\epsilon}=w^{\epsilon}=0 \text { in } \mathcal{Q}_{0}^{\epsilon}:=\left\{(x, T) \in \mathcal{Q}: x \geq \ell^{\epsilon}(T)\right\}
$$

with $\ell^{\epsilon}(0)=0$.

\subsubsection{The equations of motion}

The motion of the debonded part of the film is governed by the (classical) wave equation, of [10]:

$$
\frac{\partial^{2} w^{\epsilon}}{\partial x^{2}}-\epsilon^{2} \frac{\partial^{2} w^{\epsilon}}{\partial T^{2}}=0 \quad \text { in } \quad \mathcal{Q} \backslash \mathcal{Q}_{0}^{\epsilon}
$$

Let us note that the wave velocity in the $(x, T)$-plane is equal to $1 / \epsilon$ because of the time rescaling and the use of dimensionless coordinates. Moreover, since the gradient of the displacement is discontinuous across some curves of the $(x, T)$-plane, it is better to set the problem in terms of the gradient components. Thus, let us denote by $\omega^{\epsilon}$ and $v^{\epsilon}$ the partial derivatives of $w^{\epsilon}$ :

$$
\omega^{\epsilon}=\frac{\partial w^{\epsilon}}{\partial x}, \quad v^{\epsilon}=\frac{\partial w^{\epsilon}}{\partial T} .
$$

The field $\omega^{\epsilon}$ represents the infinitesimal rotation of the film and the field $v^{\epsilon}$ is the rescaled transverse velocity of the film (the real transverse velocity of the material points of the film is equal to $\epsilon c v^{\epsilon}$ because of the time rescaling).

The rescaled transverse velocity must satisfy the boundary condition

$$
v^{\epsilon}(0, T)=1, \forall T>0 .
$$

Both fields have to satisfy the Hadamard compatibility condition and the equation of motion

$$
0=\frac{\partial \omega^{\epsilon}}{\partial T}-\frac{\partial v^{\epsilon}}{\partial x}, \quad 0=\frac{\partial \omega^{\epsilon}}{\partial x}-\epsilon^{2} \frac{\partial v^{\epsilon}}{\partial T} .
$$

Let $S^{\epsilon}$ be the set of points $(x, T) \in \mathcal{Q} \backslash \mathcal{Q}_{0}^{\epsilon}$ where $\omega^{\epsilon}$ and $v^{\epsilon}$ are discontinuous; that corresponds to the shock waves. The equations (9) are to be satisfied in $\mathcal{Q} \backslash\left(S^{\epsilon} \cup \mathcal{Q}_{0}^{\epsilon}\right)$. On $S^{\epsilon}$, they have to be replaced by the Hadamard compatibility condition and the Rankine-Hugoniot condition, see [11]:

$$
0=s \llbracket \omega^{\epsilon} \rrbracket+\llbracket v^{\epsilon} \rrbracket, \quad 0=\llbracket \omega^{\epsilon} \rrbracket+s \epsilon^{2} \llbracket v^{\epsilon} \rrbracket .
$$

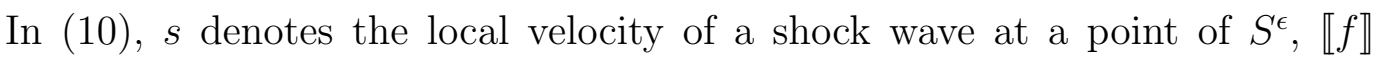
denotes the jump discontinuity of the function $f$ across $S^{\epsilon}$ :

$$
\llbracket f \rrbracket=f_{+}-f_{-} \quad \text { on } \quad S^{\epsilon},
$$


$f_{+}$and $f_{-}$denoting respectively the limit after and before the passage of the wave. We deduce from (10) that $s^{2} \epsilon^{2}=1$, i.e. , the shock waves propagate with a velocity equal to $\pm 1 / \epsilon$ (in the $(x, T)$-plane). Accordingly, we can divide $S^{\epsilon}$ into two subsets: the forward shock waves $S_{+}^{\epsilon}$ where $s=+1 / \epsilon$ and the backward shock waves $S_{-}^{\epsilon}$ where $s=-1 / \epsilon$. Then, the only jump condition which remains to satisfy is the Hadamard compatibility condition which becomes:

$$
0=\llbracket \omega^{\epsilon} \rrbracket+\epsilon \llbracket v^{\epsilon} \rrbracket \text { on } S_{+}^{\epsilon}, \quad 0=\llbracket \omega^{\epsilon} \rrbracket-\epsilon \llbracket v^{\epsilon} \rrbracket \text { on } S_{-}^{\epsilon} .
$$

\subsubsection{Griffith's law of debonding}

Let us denote by $\Gamma^{\epsilon}$ the front of debonding and $\dot{\ell}^{\epsilon}$ its local speed in the $(x, T)$ plane:

$$
\Gamma^{\epsilon}:=\left\{\left(\ell^{\epsilon}(T), T\right): T>0\right\}, \quad \dot{\ell}^{\epsilon}=\frac{d \ell^{\epsilon}}{d T} .
$$

The displacement $w^{\epsilon}$ is continuous on $\Gamma^{\epsilon}$ (and equal to 0 ), but $\omega^{\epsilon}$ and $v^{\epsilon}$ are discontinuous and must satisfy the Hadamard compatibility condition

$$
0=\llbracket v^{\epsilon} \rrbracket+\dot{\ell}^{\epsilon} \llbracket \omega^{\epsilon} \rrbracket \text { on } \Gamma^{\epsilon} .
$$

In (14) the double brackets denote, like in (11), the difference between the limit after and the limit before the passage of the front of debonding. The debonding of the film is governed by Griffith's law [9] which is is formulated in terms of the dynamic energy release rate $G^{\epsilon}$. In a general two-dimensional context, $G^{\epsilon}$ is defined as the limit of a path integral where the path tends to the tip of the crack, see [12]. In our one-dimensional context, $G^{\epsilon}$ is given by

$$
G^{\epsilon}=\frac{N}{2}\left(\llbracket \omega^{\epsilon 2} \rrbracket-\epsilon^{2} \llbracket v^{\epsilon 2} \rrbracket\right)
$$

where the jumps are defined on $\Gamma^{\epsilon}$, cf the appendix. Therefore, since the limits before the passage of the debonding front vanish, upon using (14), $G^{\epsilon}$ reads as

$$
G^{\epsilon}=\frac{N}{2}\left(1-\left(\epsilon \dot{\ell}^{\epsilon}\right)^{2}\right)\left(\omega_{+}^{\epsilon}\right)^{2} .
$$

Denoting by $x \mapsto G_{c}(x)$ the spatial repartition of the toughness, the Griffith law reads as, cf $[9,13]$ :

$$
\dot{\ell}^{\epsilon} \geq 0, \quad G^{\epsilon} \leq G_{c}\left(\ell^{\epsilon}\right), \quad\left(G^{\epsilon}-G_{c}\left(\ell^{\epsilon}\right)\right) \dot{\ell}^{\epsilon}=0 .
$$

In (17) the first inequality is the irreversibility condition, the second inequality is the Griffith criterion requiring that the dynamic energy release rate must be always less than the local toughness $G_{c}(\ell)$, and the last equality stipulates that the debonding evolves only when the dynamic energy release rate is equal to the local toughness. According to (16) and (17), we obtain

$$
0<\epsilon \dot{\ell}^{\epsilon}<1
$$


meaning that the speed of debonding is necessarily less than the wave speed.

\subsubsection{Last definitions}

The interpretation of the solution in energetic terms is fundamental in the sequel. Let us define the various energies involved. Since the film is perfectly flexible, the bending energy is neglected and the potential energy at time $t$ is simply equal to the opposite of the work produced by the tension $N$ in the displacement $u^{\epsilon}(0, T)$. Therefore, using (4), the potential energy of the film at time $t$ can be written

$$
\mathcal{P}^{\epsilon}(T)=\frac{N L}{2} \int_{0}^{\infty}\left(\frac{\partial w^{\epsilon}}{\partial x}\right)^{2}(x, T) d x .
$$

The kinetic energy of the film at time $t$ can be read as

$$
\mathcal{K}^{\epsilon}(T)=\epsilon^{2} \frac{N L}{2} \int_{0}^{\infty}\left(\frac{\partial w^{\epsilon}}{\partial T}\right)^{2}(x, T) d x
$$

the factor $\epsilon^{2}$ being due to the rescaling (2) of the time. Following Griffith's assumption the surface energy of the film at time $t$ reads as

$$
\mathcal{S}^{\epsilon}(T)=L \int_{0}^{\ell^{\epsilon}(T)} G_{c}(x) d x
$$

To summarize this setting of the dynamic debonding evolution, the fields $\omega^{\epsilon}$, $v^{\epsilon}$ and the debonding length $\ell^{\epsilon}$ must satisfy (8), (9), (12), (14), (16)-(17). We will solve that problem in two particular cases of spatial toughness repartition:

Case a: $\quad G_{c}(x)=\left\{\begin{array}{ll}\gamma_{1} N & \text { if } 0<x<1 \\ \gamma_{2} N & \text { if } x \geq 1\end{array}\right.$,

Case b: $\quad G_{c}(x)=\left\{\begin{array}{ll}\gamma_{2} N & \text { if } 0<x \leq 1 \\ \gamma_{1} N & \text { if } x>1\end{array}\right.$,

where in both cases $\gamma_{2}>\gamma_{1}>0$.

Remark 1 We construct in each case a (particular) solution. The issue of the uniqueness will not be discussed in this paper.

\subsection{The quasi-static approach}

In the quasi-static approach, the kinetic energy is neglected as well as any inertial effect and the film is assumed to be at equilibrium for every value of 
the given opening $T$. Therefore, let $T$ and $\ell$ be the given opening and the debonding length. By virtue of the theorem of potential energy minimum, the transverse displacement field at equilibrium, $w^{0}$, is that which minimizes the potential energy on all kinematically admissible vertical displacement fields $w$. Since $w$ is admissible if and only if $w \geq 0, w=0$ in $[\ell,+\infty)$ and $w(0)=T$, and, since the associated potential energy reads as $\int_{0}^{\ell} N L w^{\prime}(x)^{2} / 2 d x$, then the potential energy of the film at equilibrium, $\mathcal{P}(T, \ell)$, is given by

$$
\mathcal{P}(T, \ell)=\min _{\{w \geq 0: w(0)=T\}} \int_{0}^{\ell} \frac{N L}{2} w^{\prime}(x)^{2} d x .
$$

In (24) and above the prime denotes the derivative with respect to $x$. Then we easily deduce that the minimizer $w^{0}$ is given by

$$
w^{0}(x)=T\left(1-\frac{x}{\ell}\right)^{+}
$$

the superscript + denoting the positive part. Therefore the potential energy and the surface energy are given by

$$
\mathcal{P}(T, \ell)=\frac{N L T^{2}}{2 \ell}, \quad \mathcal{S}(\ell)=L \int_{0}^{\ell} G_{c}(x) d x
$$

and the quasi-static potential energy release rate reads as

$$
G:=-\frac{1}{L} \frac{\partial \mathcal{P}}{\partial \ell}(T, \ell)=\frac{N T^{2}}{2 \ell^{2}}
$$

Let us note that $\mathcal{P}$ is a strictly convex function of $\ell$ at given $T>0$, while $\mathcal{S}$ is a convex function of $\ell$ if and only if $x \mapsto G_{c}(x)$ is monotone increasing. Therefore, the total energy $\mathcal{E}(T, \ell):=\mathcal{P}(T, \ell)+\mathcal{S}(\ell)$ is a strictly convex function of $\ell$ at given $T>0$ in Case a, but not in Case b.

If we still assume that the debonding evolution is governed by Griffith's law, then the function $T \mapsto \ell(T)$ giving the evolution of the length debonding must be an absolutely continuous function (see [14] for a precise definition of absolutely continuous functions) which satisfies, for almost all $T>0$ :

$$
\dot{\ell}(T) \geq 0, \quad \frac{N T^{2}}{2 \ell(T)^{2}} \leq G_{c}(\ell(T)), \quad\left(\frac{N T^{2}}{2 \ell(T)^{2}}-G_{c}(\ell(T))\right) \dot{\ell}(T)=0,
$$

with the initial condition $\ell(0)=0$. We have so the fundamental result

Proposition 2.1 In Case a, Griffith's law (28) admits the solution given by

$$
\ell(T)= \begin{cases}T / \sqrt{2 \gamma_{1}}, & \text { if } 0 \leq T \leq \sqrt{2 \gamma_{1}} \\ 1, & \text { if } \sqrt{2 \gamma_{1}} \leq T \leq \sqrt{2 \gamma_{2}} . \\ T / \sqrt{2 \gamma_{2}}, & \text { if } T \geq \sqrt{2 \gamma_{2}}\end{cases}
$$


In $\mathbf{C a s e} \mathbf{b}$ there is no (absolutely continuous) solution.

Proof. In Case a let us verify that $T \mapsto \ell(T)$ given by (29) is a solution of (28). Its continuity and its monotonicity are easily checked. We have $G(T)=$ $\gamma_{1} N=G_{c}(\ell(T))$ for $T \in\left(0, \sqrt{2 \gamma_{1}}\right)$ and $G(T)=\gamma_{2} N=G_{c}(\ell(T))$ for $T \geq \sqrt{2 \gamma_{2}}$. For $T \in\left(\sqrt{2 \gamma_{1}}, \sqrt{2 \gamma_{2}}\right)$, we have $G(T)<G_{c}(\ell(T))=\gamma_{2} N$ and $\dot{\ell}(T)=0$.

Let us consider Case $\mathbf{b}$ and assume that there exists an absolutely continuous solution $T \mapsto \ell(T)$. Then $\ell(T)$ grows from 0 to $\infty$ when $T$ grows from 0 to $+\infty$. (Indeed, assume the contrary and set $\ell_{M}=\lim _{T \rightarrow \infty} \ell(T)<\infty$. Then, according to $(28)_{2}$, we should have $T^{2} \leq 2 \gamma_{2} \ell_{M}, \forall T>0$, what is impossible. Hence $\lim _{T \rightarrow \infty} \ell(T)=\infty$.) Therefore, $\ell(T)=1$ for some $T$. Moreover, because of the monotonicity of $T \mapsto \ell(T)$, there exist $T_{0}$ and $T_{1}$ with $0<T_{0} \leq T_{1}<$ $+\infty$ such that $\ell(T)<1$ for $T<T_{0}, \ell(T)=1$ for $T \in\left[T_{0}, T_{1}\right]$ and $\ell(T)>1$ for $T>T_{1}$. We should have $G\left(T_{0}\right)=\gamma_{2} N$. (Indeed, if not, $G\left(T_{0}\right)<\gamma_{2} N$ and by continuity $G(T)<\gamma_{2} N$ in some interval $\left(T_{0}-h, T_{0}\right]$. Hence, according to $(28)_{3}$ and by the absolute continuity assumption, $\ell(T)=1$ in that interval, what is in contradiction with the definition of $T_{0}$.) But, we should have also $G\left(T_{1}\right) \leq \gamma_{1} N$. (Indeed, $G(T) \leq G_{c}(\ell(T))=\gamma_{1} N$ for $T>T_{1}$ and hence by continuity the inequality holds at $T_{1}$.) Finally, we should have $2 \gamma_{2}=T_{0}^{2} \leq T_{1}^{2} \leq 2 \gamma_{1}$, which is in contradiction with the assumption on the toughness. Accordingly, there is no absolutely continuous solution.

In Case a, a more careful analysis could show that (29) is the unique (absolutely continuous) solution of $(28)$ and that $\ell(T)$ is, at every $T$, the global minimizer of $l \mapsto \mathcal{E}(T, l)$ on $\{l \geq 0\}$. The proof is not reproduced here, but the interested reader is referred to [15] for similar results relating global minimization to Griffith's law. The graph of $T \mapsto \ell(T)$ is plotted in Figure 2. The

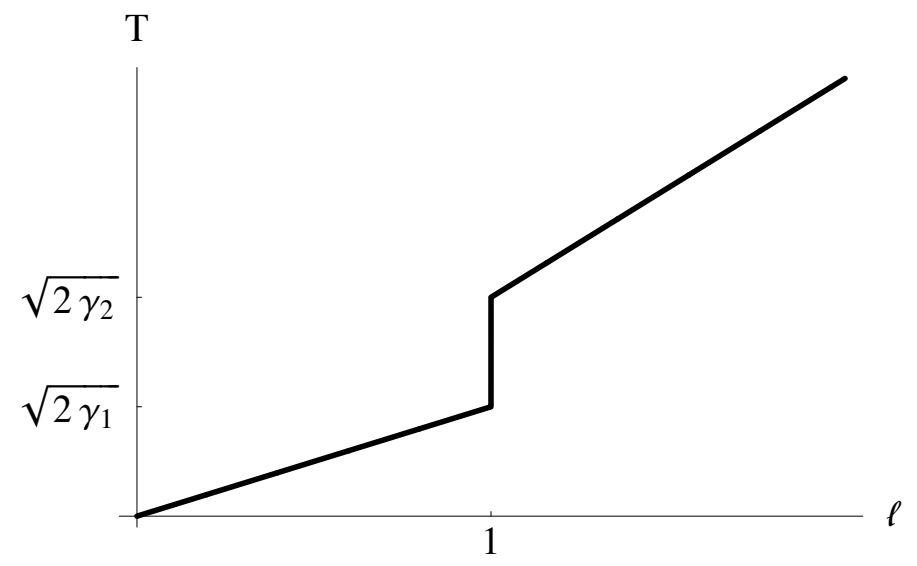

Fig. 2. The quasi-static evolution of the debonding in Case a by using Griffith's law. 
evolution of the potential and surface energies are given by

$$
\mathcal{P}(T)= \begin{cases}\sqrt{2 \gamma_{1}} T N L / 2 & \text { if } T \leq \sqrt{2 \gamma_{1}} \\ T^{2} N L / 2 & \text { if } \sqrt{2 \gamma_{1}} \leq T \leq \sqrt{2 \gamma_{2}} \\ \sqrt{2 \gamma_{2}} T N L / 2 & \text { if } T \geq \sqrt{2 \gamma_{2}}\end{cases}
$$

and

$$
\mathcal{S}(T)= \begin{cases}\sqrt{2 \gamma_{1}} T N L / 2 & \text { if } T \leq \sqrt{2 \gamma_{1}} \\ \gamma_{1} N L & \text { if } \sqrt{2 \gamma_{1}} \leq T \leq \sqrt{2 \gamma_{2}} \\ \left(\gamma_{1}-\gamma_{2}+\sqrt{2 \gamma_{2}} T / 2\right) N L & \text { if } T \geq \sqrt{2 \gamma_{2}}\end{cases}
$$

In Case b, the debonding evolution is necessarily discontinuous. We can expect that the debonding evolves continuously and follows Griffith's law until the debonding reaches the point $x=1$ where there is a loss of toughness. In other words, we can expect that $\ell(T)=T / \sqrt{2 \gamma_{2}}$ for $T \in\left[0, \sqrt{2 \gamma_{2}}\right)$. As it is shown in the proof of Proposition 2.1, at $T=\sqrt{2 \gamma_{2}}, \ell$ must jump from 1 to a certain $\ell_{c}>1$. This phase of instability of the debonding can be interpreted from the evolution of the graph of the total energy as a function of the debonding length when the given opening grows, cf Figure 3 and see also [16] for the use of the concepts of instability in Fracture Mechanics. For $T<\sqrt{2 \gamma_{2}}$, $\ell(T)=T / \sqrt{2 \gamma_{2}}$ is a local minimum of $l \mapsto \mathcal{E}(T, l)$. But at $T=\sqrt{2 \gamma_{2}}$, this local minimum disappears and the debonding length must jump from $l=1$. The question is: "what is the equation giving the value of the jump?". Clearly, the quasi-static Griffith law is unable to give the answer because it is written only for smooth evolutions. We must introduce a new criterion and the main goal of the paper is to deduce the right equation from a dynamic analysis. Considering the graph of $l \mapsto \mathcal{E}\left(\sqrt{2 \gamma_{2}}, l\right)$ and by analogy with the motion of a ball in a basin, we could propose that the debonding length "falls" in the energy well, i.e. at the point $\ell_{m}$ such that $\partial \mathcal{E} / \partial l\left(\sqrt{2 \gamma_{2}}, \ell_{m}\right)=0$ or in other words to the point such that $G=\gamma_{1} N$. The dynamic analysis will show that this is not the good value, but that the right principle is the energy conservation: the jump of the debonding is such that the total quasi-static energy just after the jump remains equal to that just before the jump. Let us assume that this rule holds. Then, the length debonding after the jump, $\ell_{c}$, is such that $\ell_{c}>1$ and $\mathcal{E}\left(\sqrt{2 \gamma_{2}}, 1\right)=\mathcal{E}\left(\sqrt{2 \gamma_{2}}, \ell_{c}\right)$. A direct calculation gives

$$
\ell_{c}=\frac{\gamma_{2}}{\gamma_{1}}>\ell_{m}=\sqrt{\frac{\gamma_{2}}{\gamma_{1}}}
$$

After this jump, the debonding front will propagate in a medium with a constant toughness and we can assume that the debonding evolution will follow the quasi-static Griffith law again. Since $\ell_{c}>\ell_{m}$, the energy release rate just after the jump is less than $\gamma_{1} N$ and the debonding length will remain at its value $\ell_{c}$ as long as the opening $T$ is not sufficiently large so that the energy 

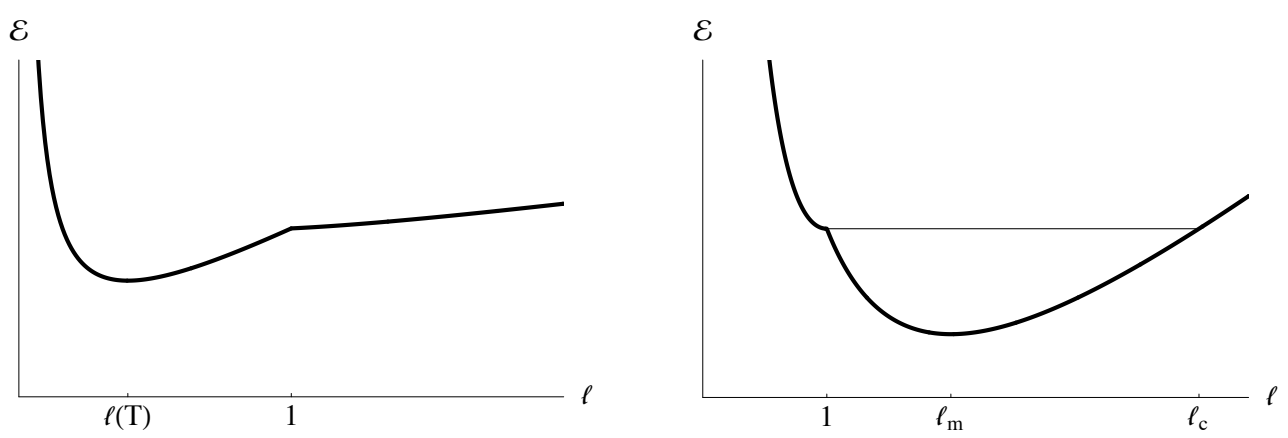

Fig. 3. The graph of the total energy as a function of the debonding length $\ell$ for a given opening $T$ in Case b. left: when $T<\sqrt{2 \gamma_{2}}$; right: when $T=\sqrt{2 \gamma_{2}}$ and the value $\ell_{c}$ of the debonding length after the jump given by the energy conservation principle.

release rate be equal to $\gamma_{1} N$. That holds as long as $T \leq T_{c}$ with $T_{c}$ given by

$$
T_{c}=\frac{2 \gamma_{2}}{\sqrt{2 \gamma_{1}}}
$$

Then, when $T>T_{c}$, the debonding restarts and the debonding length grows so that the energy release rate remains equal to $\gamma_{1} N$. Therefore, $\ell(T)=T / \sqrt{2 \gamma_{1}}$ for $T>T_{c}$.

To summarize this analysis of Case $\mathbf{b}$, if we assume that the debonding evolution follows always Griffith's law except during the unstable phase where the jump is governed by the energy conservation principle, the graph of $T \mapsto \ell(T)$ should be that plotted in Figure 4,

$$
\ell(T)= \begin{cases}T / \sqrt{2 \gamma_{2}} & \text { if } 0 \leq T<\sqrt{2 \gamma_{2}} \\ \gamma_{2} / \gamma_{1} & \text { if } \sqrt{2 \gamma_{2}}<T \leq 2 \gamma_{2} / \sqrt{2 \gamma_{1}} \\ T / \sqrt{2 \gamma_{1}} & \text { if } T \geq 2 \gamma_{2} / \sqrt{2 \gamma_{1}}\end{cases}
$$

The main goal of the paper is to justify this conjecture by solving the full dynamic problem and by passing to the limit when the speed of loading goes down to 0 . We will also state a result of convergence for the energies. Their values corresponding to the conjectured quasi-static solution are given by

$$
\mathcal{P}(T)= \begin{cases}\sqrt{2 \gamma_{2}} T N L / 2 & \text { if } T<\sqrt{2 \gamma_{2}} \\ T^{2} N L / 2 \ell_{c} & \text { if } \sqrt{2 \gamma_{2}}<T \leq T_{c} \\ \sqrt{2 \gamma_{1}} T N L / 2 & \text { if } T \geq T_{c}\end{cases}
$$

and

$$
\mathcal{S}(T)= \begin{cases}\sqrt{2 \gamma_{2}} T N L / 2 & \text { if } T<\sqrt{2 \gamma_{2}} \\ \left(2 \gamma_{2}-\gamma_{1}\right) N L & \text { if } \sqrt{2 \gamma_{2}}<T \leq T_{c} \\ \left(\gamma_{2}-\gamma_{1}+\sqrt{2 \gamma_{1}} T / 2\right) N L & \text { if } T \geq T_{c}\end{cases}
$$




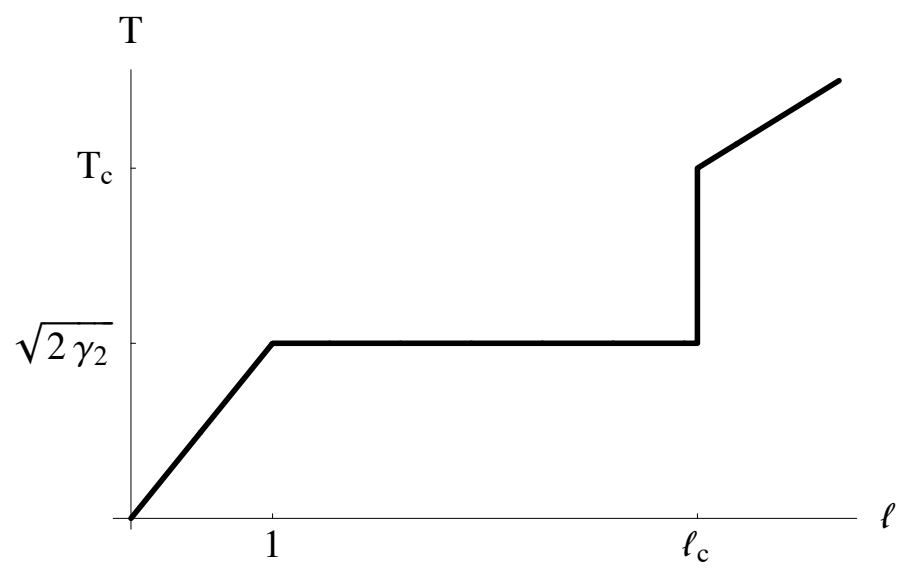

Fig. 4. The quasi-static evolution of the debonding in Case $\mathbf{b}$ by using both Griffith's law for the continuous phases and the principle of energy conservation for the jump.

Let us remark that their respective values just before and just after the jump are given by

$$
\begin{gathered}
\mathcal{P}^{-}\left(\sqrt{2 \gamma_{2}}\right)=\gamma_{2} N L, \quad \mathcal{P}^{+}\left(\sqrt{2 \gamma_{2}}\right)=\gamma_{1} N L, \\
\mathcal{S}^{-}\left(\sqrt{2 \gamma_{2}}\right)=\gamma_{2} N L, \quad \mathcal{S}^{+}\left(\sqrt{2 \gamma_{2}}\right)=\left(2 \gamma_{2}-\gamma_{1}\right) N L .
\end{gathered}
$$

Of course, since the total energy is conserved, all the released potential energy is so transformed into surface energy.

\section{The dynamic analysis}

\subsection{The structure of the dynamic solution}

The structure of the dynamic solution is the same for each case. The solution contains a first phase of debonding evolution where the debonding front propagates at a constant speed until it reaches the point $x=1$ where the toughness changes. That change of the toughness generates a backward (traveling) shock wave as well as a change of the speed of debonding. The backward shock wave is reflected at $x=0$ and is transformed into a forward (traveling) shock wave. Since the wave speed is necessarily greater than the speed of debonding, the forward shock wave intersects the front of debonding. Then, the speed of debonding changes again and the forward shock wave is transformed into a backward shock wave. This second backward shock wave is reflected itself at $x=0$ into a forward shock wave, which in its turn will intersect the front of debonding. Then, the speed of debonding changes, a backward shock wave is generated and so on.

We seek for a solution for which the speed of debonding as well as the rota- 


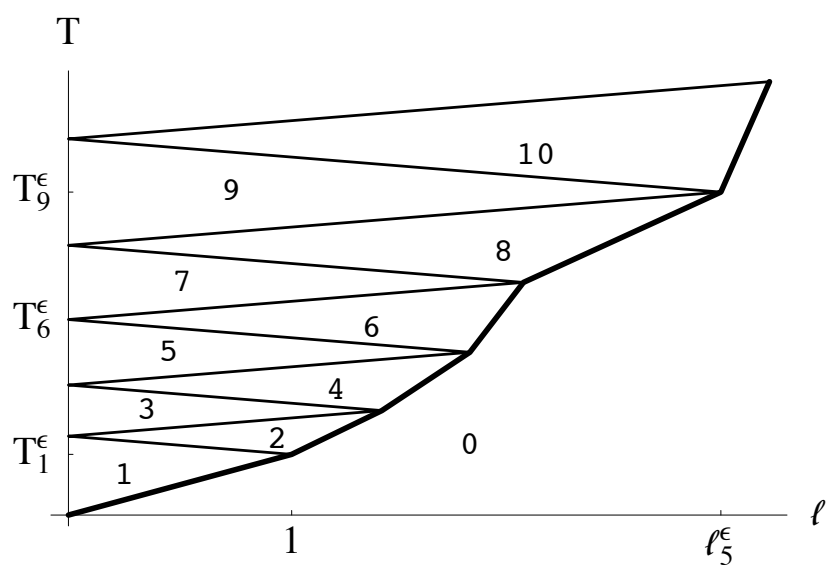

Fig. 5. Structure of the dynamic solution and numbering of the sectors. Thick line: debonding front; thin lines: backward and forward shock waves.

tion and the velocity fields are piecewise constant, i.e. constant in each sector delimited by the backward shock wave, the forward shock wave and the front of debonding, see Figure 5. Those sectors are denoted by $\left\{\mathcal{Q}_{i}^{\epsilon}\right\}_{i \in \mathbb{N}}$. The sector $\mathcal{Q}_{0}^{\epsilon}$ corresponds to the sector ahead of the front of debonding, i.e. where $\omega^{\epsilon}=v^{\epsilon}=0$. The sector $\mathcal{Q}_{1}^{\epsilon}$ is delimited by the front of debonding before $x=1$, $\Gamma_{0}^{\epsilon}$, the backward shock wave generated at $x=1, S_{1}^{\epsilon}$, and the boundary $x=0$. Then the index $i$ of the sector is incremented at each reflection of the shock wave. In the sector $\mathcal{Q}_{i}^{\epsilon}, i \geq 0$, the (constant) values of $\left(\omega^{\epsilon}, v^{\epsilon}\right)$ are denoted $\left(\omega_{i}^{\epsilon}, v_{i}^{\epsilon}\right)$. Thus, $\omega_{0}^{\epsilon}=v_{0}^{\epsilon}=0$. The speed of debonding before $x<1$, that is the slope of the line segment $\Gamma_{0}^{\epsilon}$ separating $\mathcal{Q}_{0}^{\epsilon}$ and $\mathcal{Q}_{1}^{\epsilon}$, is denoted by $\dot{\ell}_{0}^{\epsilon}$ and the associated dynamic energy release rate by $G_{0}^{\epsilon}$. The front of debonding reaches $x=1$ at $T=T_{1}^{\epsilon}$. Once the debonding propagates in the part $x>1$, the speed of debonding and the energy release rate on the line segment $\Gamma_{i}^{\epsilon}$ separating $\mathcal{Q}_{0}^{\epsilon}$ and $\mathcal{Q}_{2 i}^{\epsilon}, i \geq 1$, are denoted by $\dot{\ell}_{i}^{\epsilon}$ and $G_{i}^{\epsilon}$. The values of the opening when the backward shock wave is reflected at $x=0$ and is transformed into a forward shock wave are denoted $T_{2 i}^{\epsilon}, i \geq 1$. The values of the opening when the forward shock wave intersects the front of debonding and is transformed into a backward shock wave are denoted $T_{2 i-1}^{\epsilon}, i \geq 2$. The associated position of the debonding front is denoted $\ell_{i}^{\epsilon}$.

We put $T_{0}^{\epsilon}=0$ and $\ell_{1}^{\epsilon}=1$. The equation of the first front of propagation reads as

$$
\Gamma_{0}^{\epsilon}: l=\dot{\ell}_{0}^{\epsilon} T, 0<T<T_{1}^{\epsilon} .
$$

The equations of backward shock wave $\mathcal{S}_{2 i-1}^{\epsilon}$ between $\mathcal{Q}_{2 i-1}^{\epsilon}$ and $\mathcal{Q}_{2 i}^{\epsilon}$, and of the forward shock wave $\mathcal{S}_{2 i}^{\epsilon}$ between $\mathcal{Q}_{2 i}^{\epsilon}$ and $\mathcal{Q}_{2 i+1}^{\epsilon}$ can be written as

$$
\mathcal{S}_{2 i-1}^{\epsilon}: T=T_{2 i}^{\epsilon}-\epsilon l, 0<l<\ell_{i}^{\epsilon}, \quad \mathcal{S}_{2 i}^{\epsilon}: T=T_{2 i}^{\epsilon}+\epsilon l, 0<l<\ell_{i+1}^{\epsilon},
$$

whereas the equation of $\Gamma_{i}^{\epsilon}$, for $i \geq 1$ reads as

$$
\Gamma_{i}^{\epsilon}: l=\ell_{i}^{\epsilon}+\dot{\ell}_{i}^{\epsilon}\left(T-T_{2 i-1}^{\epsilon}\right), T_{2 i-1}^{\epsilon} \leq T<T_{2 i+1}^{\epsilon} .
$$


Therefore

$$
S^{\epsilon}=\bigcup_{i \geq 1} \mathcal{S}_{i}^{\epsilon}, \quad \Gamma^{\epsilon}=\bigcup_{i \in \mathbb{N}} \Gamma_{i}^{\epsilon}
$$

and the $T_{i}^{\epsilon}$ 's are related to the $\ell_{i}^{\epsilon}$ 's by

$$
T_{2 i}^{\epsilon}-T_{2 i-1}^{\epsilon}=\epsilon \ell_{i}^{\epsilon}, \quad T_{2 i+1}^{\epsilon}-T_{2 i}^{\epsilon}=\epsilon \ell_{i+1}^{\epsilon}, \quad \forall i \geq 1
$$

Since the fields are piecewise constant, (9) is automatically satisfied in $\mathcal{Q} \backslash\left(S^{\epsilon} \cup\right.$ $\left.\Gamma^{\epsilon}\right)$. It remains to find the sequences $\omega_{i}^{\epsilon}, v_{i}^{\epsilon}$ and $\dot{\ell}_{i}^{\epsilon}$ for $i \in \mathbb{N}$. The procedure is the same in both cases and we use in this subsection the following notation for the toughness

$$
G_{c}(x)= \begin{cases}\gamma_{-} N & \text { if } x<1 \\ \gamma_{+} N & \text { if } x>1\end{cases}
$$

Let us begin by the first phase of debonding. The boundary condition $(8)_{1}$ gives $v_{1}^{\epsilon}=1$ and (14) leads to $\dot{\ell}_{0}^{\epsilon} \omega_{1}^{\epsilon}=-1$. Hence $\dot{\ell}_{0}^{\epsilon} \neq 0$ and, according to $(17)_{3}, G_{0}^{\epsilon}=\gamma_{-} N$. Using (16), we get $\dot{\ell}_{0}^{\epsilon 2}=\left(2 \gamma_{-}+\epsilon^{2}\right)^{-1}$. Since $\dot{\ell}_{0}^{\epsilon}>0$ by virtue of $(17)_{1}$, we finally have

$$
\dot{\ell}_{0}^{\epsilon}=\frac{1}{\sqrt{2 \gamma_{-}+\epsilon^{2}}}, \quad \omega_{1}^{\epsilon}=-\sqrt{2 \gamma_{-}+\epsilon^{2}}, \quad v_{1}^{\epsilon}=1 .
$$

To find the debonding evolution in the part $x>1$, we use the following statement:

Proposition 3.1 Let $i \geq 1$. In $\mathcal{Q}_{2 i-1}^{\epsilon}, v_{2 i-1}^{\epsilon}=1$; let us assume that $\omega_{2 i-1}^{\epsilon}$ and $\ell_{i}^{\epsilon}$ are known. Then $\omega_{2 i}^{\epsilon}, v_{2 i}^{\epsilon}$ and $\dot{\ell}_{i}^{\epsilon}$ are given by

$$
\dot{\ell}_{i}^{\epsilon}=\frac{1}{\epsilon} \frac{\left(\left(\omega_{2 i-1}^{\epsilon}-\epsilon\right)^{2}-2 \gamma_{+}\right)^{+}}{\left(\omega_{2 i-1}^{\epsilon}-\epsilon\right)^{2}+2 \gamma_{+}}, \quad \omega_{2 i}^{\epsilon}=\frac{\omega_{2 i-1}^{\epsilon}-\epsilon}{1+\epsilon \dot{\ell}_{i}^{\epsilon}}, \quad v_{2 i}^{\epsilon}=-\dot{\ell}_{i}^{\epsilon} \omega_{2 i}^{\epsilon},
$$

the superscript + in the first relation denoting the positive part. Furthermore, $\omega_{2 i+1}^{\epsilon}, v_{2 i+1}^{\epsilon}$ and $\ell_{i+1}^{\epsilon}$ are given by

$$
\omega_{2 i+1}^{\epsilon}=2 \omega_{2 i}^{\epsilon}-\omega_{2 i-1}^{\epsilon}, \quad v_{2 i+1}^{\epsilon}=1, \quad \ell_{i+1}^{\epsilon}=\frac{1+\epsilon \dot{\ell}_{i}^{\epsilon}}{1-\epsilon \dot{\ell}_{i}^{\epsilon}} \ell_{i}^{\epsilon} .
$$

Moreover the different energies are piecewise linear function of $T$. Their values at $T_{i}^{\epsilon}$ are given by 


$$
\begin{aligned}
\mathcal{P}^{\epsilon}\left(T_{2 i-1}^{\epsilon}\right) & =\frac{N L}{2} \omega_{2 i-1}^{\epsilon} \ell_{i}^{\epsilon}, \\
\mathcal{K}^{\epsilon}\left(T_{2 i-1}^{\epsilon}\right) & =\frac{\epsilon^{2} N L}{2} \ell_{i}^{\epsilon}, \\
\mathcal{S}\left(T_{2 i-1}^{\epsilon}\right) & =\left(\gamma_{-}+\gamma_{+}\left(\ell_{i}^{\epsilon}-1\right)\right) N L, \\
\mathcal{P}^{\epsilon}\left(T_{2 i}^{\epsilon}\right) & =\frac{N L}{2} \omega_{2 i}^{\epsilon}{ }^{2}\left(1+\epsilon \dot{\ell}_{i}^{\epsilon}\right) \ell_{i}^{\epsilon}, \\
\mathcal{K}^{\epsilon}\left(T_{2 i}^{\epsilon}\right) & =\frac{\epsilon^{2} N L}{2} v_{2 i}^{\epsilon}{ }^{2}\left(1+\epsilon \dot{\ell}_{i}^{\epsilon}\right) \ell_{i}^{\epsilon}, \\
\mathcal{S}\left(T_{2 i}^{\epsilon}\right) & =\left(\gamma_{-}+\gamma_{+}\left(\left(1+\epsilon \dot{\ell}_{i}^{\epsilon}\right) \ell_{i}^{\epsilon}-1\right)\right) N L .
\end{aligned}
$$

Proof. Most of the above relations are direct consequences of the boundary condition $(8)_{1}$, the Hadamard compatibility conditions (12) and (14) or the geometric properties (40), (41) and (43). In particular, $(46)_{2}$ is obtained by a simple combination of (12) and (14). The only part which needs a careful analysis is the formula $(46)_{1}$ for $\dot{\ell}_{i}^{\epsilon}$. Let us first use (14) and (16) to obtain

$$
v_{2 i}^{\epsilon}=-\dot{\ell}_{i}^{\epsilon} \omega_{2 i}^{\epsilon}, \quad G_{i}^{\epsilon}=\frac{N}{2}\left(1-\epsilon^{2} \dot{\ell}_{i}^{\epsilon 2}\right) \omega_{2 i}^{\epsilon} .
$$

According to (17), if $G_{i}^{\epsilon}<\gamma_{+} N$ then $\dot{\ell}_{i}^{\epsilon}=0$. In such a case, because of (54), $\left|\omega_{2 i}^{\epsilon}\right|<\sqrt{2 \gamma_{+}}$. Otherwise, if $G_{i}^{\epsilon}=\gamma_{+} N$, then, because of (54) again, we have

$$
\left|\omega_{2 i}^{\epsilon}\right| \geq \sqrt{1-\epsilon^{2} \dot{\ell}_{i}^{\epsilon 2}}\left|\omega_{2 i}^{\epsilon}\right|=\sqrt{2 \gamma_{+}}
$$

Therefore, it is sufficient to compare $\left|\omega_{2 i}^{\epsilon}\right|$ to $\sqrt{2 \gamma_{+}}$to determine whether the debonding grows and find $\dot{\ell}_{i}^{\epsilon}$. But, according to $(46)_{2},\left|\omega_{2 i}^{\epsilon}\right| \geq \sqrt{2 \gamma_{+}}$is equivalent to $\left|\omega_{2 i-1}^{\epsilon}\right|+\epsilon \geq \sqrt{2 \gamma_{+}}$. Inserting the relationship (46) $)_{2}$ giving $\omega_{2 i}^{\epsilon}$ in terms of $\dot{\ell}_{i}^{\epsilon}$ and $\omega_{2 i-1}^{\epsilon}$ into the equation $G_{i}^{\epsilon}=\gamma_{+} N$, we finally obtain $\dot{\ell}_{i}^{\epsilon}$ in terms of $\omega_{2 i-1}^{\epsilon}$.

The energies are piecewise linear functions of $T$ because $\omega^{\epsilon}, v^{\epsilon}$ and $\dot{\ell}^{\epsilon}$ are piecewise constant. By using their definition (19), (20) and (21), and by remarking that $\ell^{\epsilon}\left(T_{2 i}^{\epsilon}\right)=\left(1+\epsilon \dot{\ell}_{i}^{\epsilon}\right) \ell_{i}^{\epsilon}$ leads to $(48)-(53)$.

\subsection{Case a}

\subsubsection{The dynamic solution}

The speed of loading $\epsilon$ is assumed to be small enough to remove some particular cases. We can distinguish three phases in the debonding evolution, cf Figure 6. 


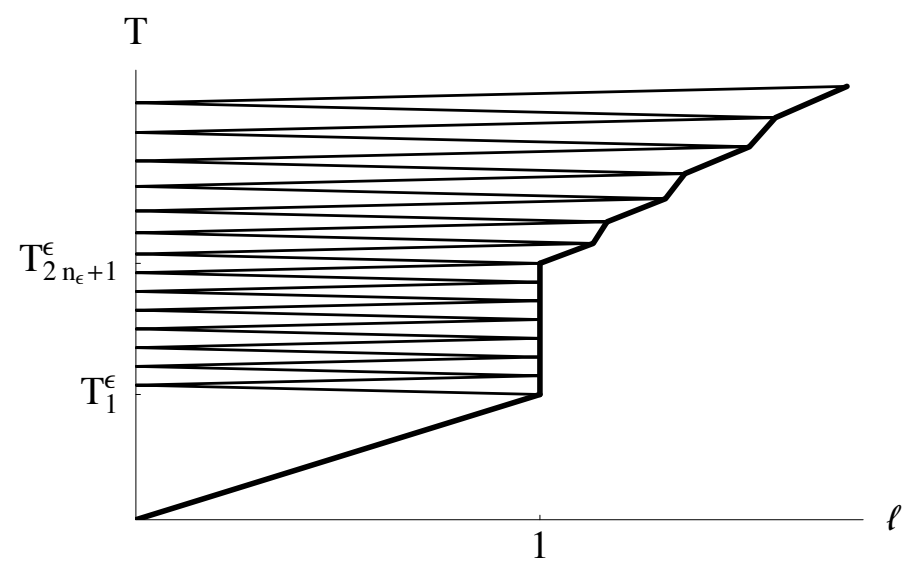

Fig. 6. Case a : Dynamic solution with the three phases of the debonding propagation for $\gamma_{1}=0.5, \gamma_{2}=2$. and $\epsilon=0.075$

3.2.1.1 Debonding in the zone with the lower toughness. In the sector $\mathcal{Q}_{1}^{\epsilon}$, we can use (45) with $\gamma_{-}=\gamma_{1}$ to obtain

$$
\dot{\ell}_{0}^{\epsilon}=\frac{1}{\sqrt{2 \gamma_{1}+\epsilon^{2}}}, \quad \omega_{1}^{\epsilon}=-\sqrt{2 \gamma_{1}+\epsilon^{2}}, \quad v_{1}^{\epsilon}=1 .
$$

The debonding front reaches $x=1$ at $T_{1}^{\epsilon}$ given by

$$
T_{1}^{\epsilon}=\sqrt{2 \gamma_{1}+\epsilon^{2}}
$$

Then the debonding evolution is governed by Proposition 3.1 with $\gamma_{+}=\gamma_{2}$.

3.2.1.2 Arrest at the change of toughness. For $\epsilon$ small enough, since $\left|\omega_{1}^{\epsilon}\right|+\epsilon=\sqrt{2 \gamma_{1}+\epsilon^{2}}+\epsilon<\sqrt{2 \gamma_{2}}$, we deduce from (46) that $\dot{\ell}_{1}^{\epsilon}=0$. The debonding stops at $x=1$ in the interval $\left(T_{1}^{\epsilon}, T_{1}^{\epsilon}+2 \epsilon\right)$ corresponding to the first back and forth of the shock wave. Upon inserting into (46) and (47), we deduce the values of the constants in $\mathcal{Q}_{2}^{\epsilon}$ and $\mathcal{Q}_{3}^{\epsilon}$

$$
\omega_{2}^{\epsilon}=-\sqrt{2 \gamma_{1}+\epsilon^{2}}-\epsilon, \quad v_{2}^{\epsilon}=0, \quad \omega_{3}^{\epsilon}=-\sqrt{2 \gamma_{1}+\epsilon^{2}}-2 \epsilon, \quad v_{3}^{\epsilon}=1 .
$$

Let us show that the debonding does not evolve during a certain number $n^{\epsilon}$ of back and forth of the shock wave. Specifically, let us show by induction that, if $1 \leq i \leq n^{\epsilon}$, with $n^{\epsilon}$ to be determined, then $\dot{\ell}_{i}^{\epsilon}=0$ and

$\omega_{2 i}^{\epsilon}=-\sqrt{2 \gamma_{1}+\epsilon^{2}}-(2 i-1) \epsilon, \quad v_{2 i}^{\epsilon}=0, \quad \omega_{2 i+1}^{\epsilon}=-\sqrt{2 \gamma_{1}+\epsilon^{2}}-2 i \epsilon \quad v_{2 i+1}^{\epsilon}=1$.

That holds for $i=1$. Let us assume that it is true until $i-1 \geq 1$ and let us find under which condition that remains true for $i$. Using the induction assumption, we have $\left|\omega_{2 i-1}^{\epsilon}\right|+\epsilon=\sqrt{2 \gamma_{1}+\epsilon^{2}}+(2 i-1) \epsilon$. Therefore, because of 
(46), if $\sqrt{2 \gamma_{1}+\epsilon^{2}}+(2 i-1) \epsilon \leq \sqrt{2 \gamma_{2}}$, then $\dot{\ell}_{i}^{\epsilon}=0$ and all the other properties follow. Hence, (58) holds as long as $i \leq n^{\epsilon}$ with

$$
n^{\epsilon}=\left\langle\frac{1}{2 \epsilon}\left(\sqrt{2 \gamma_{2}}-\sqrt{2 \gamma_{1}+\epsilon^{2}}+\epsilon\right)\right\rangle,
$$

$\langle\cdot\rangle$ denoting the integer part. In terms of the opening, this phase of arrest corresponds to the interval $\left(T_{1}^{\epsilon}, T_{2 n^{\epsilon}+1}^{\epsilon}\right)$ with

$$
T_{2 n^{\epsilon}+1}^{\epsilon}=T_{1}^{\epsilon}+2 \epsilon n^{\epsilon} .
$$

Let us note also that

$$
T_{i}^{\epsilon}=-\omega_{i}^{\epsilon}=\sqrt{2 \gamma_{1}+\epsilon^{2}}+(i-1) \epsilon, \quad 1 \leq i \leq 2 n^{\epsilon}+1 .
$$

3.2.1.3 Debonding in the zone with the higher toughness. At $T=$ $T_{2 n^{\epsilon}+1}^{\epsilon}$ the debonding restarts and propagates inside the zone where the toughness is $\gamma_{2}$. To find the solution, we use Proposition 3.1 and the following Lemma

Lemma 3.2 Let $\eta$ and $X_{0}$ be such that $0<1-2 \eta<X_{0}<1$. Then the sequence $\left\{X_{i}\right\}_{i \in \mathbb{N}}$ defined by

$$
\frac{1}{X_{i+1}}=X_{i}+2 \eta
$$

remains always between $1-2 \eta$ and 1 , its generic element $X_{i}$ is given by

$$
\begin{aligned}
& \frac{1}{X_{i}+\eta+\sqrt{1+\eta^{2}}}-\frac{1}{2 \sqrt{1+\eta^{2}}} \\
= & (-1)^{i}\left(\sqrt{1+\eta^{2}}-\eta\right)^{2 i}\left(\frac{1}{X_{0}+\eta+\sqrt{1+\eta^{2}}}-\frac{1}{2 \sqrt{1+\eta^{2}}}\right)
\end{aligned}
$$

and converges, with oscillations, to $\sqrt{1+\eta^{2}}-\eta$.

Proof. Let us verify that the sequence remains between $1-2 \eta$ and 1 . By assumption, it is true for $X_{0}$. Let $i \geq 1$. If $X_{i} \in(1-2 \eta, 1)$, then

$$
1>\frac{1}{X_{i}+2 \eta}=X_{i+1}>\frac{1}{1+2 \eta}>1-2 \eta
$$

thus $X_{i+1} \in(1-2 \eta, 1)$. Let us establish (63). We first make the following change of variable: $Y_{i}=X_{i}+\sqrt{1+\eta^{2}}+\eta$. That allows to change the former sequence into

$$
\frac{1}{Y_{i+1}}=\frac{1}{\sqrt{1+\eta^{2}}+\eta}-\frac{\left(\sqrt{1+\eta^{2}}-\eta\right)^{2}}{Y_{i}},
$$


from which we easily deduce (63). Since $\sqrt{1+\eta^{2}}-\eta<1$, the term on the right hand side of (63) tends to 0 when $i$ grows to infinity and the result of convergence follows. The oscillations around the limit are due to the change of sign with $i$ in that term.

Let us set, for $j \geq 0$,

$$
X_{j}^{\epsilon}=\frac{\sqrt{2 \gamma_{2}}}{\left|\omega_{2 n^{\epsilon}+1+2 j}^{\epsilon}\right|+\epsilon}, \quad \eta_{\epsilon}=\frac{\epsilon}{\sqrt{2 \gamma_{2}}} .
$$

By definition of $n^{\epsilon}$, we have $\sqrt{2 \gamma_{2}}<\left|\omega_{2 n^{\epsilon}+1}^{\epsilon}\right|+\epsilon=\sqrt{2 \gamma_{1}+\epsilon^{2}}+\left(2 n^{\epsilon}+1\right) \epsilon \leq$ $\sqrt{2 \gamma_{2}}+2 \epsilon$ and hence, for $\epsilon$ small enough, $0<1-2 \eta_{\epsilon}<\left(1+2 \eta_{\epsilon}\right)^{-1} \leq X_{0}^{\epsilon}<1$. Furthermore, since $\dot{\ell}_{n^{\epsilon}+1}^{\epsilon}>0$, we get from (46) and (47), $1 / X_{1}^{\epsilon}=X_{0}^{\epsilon}+2 \eta_{\epsilon}$. Since $0<X_{1}^{\epsilon}<1$, we have $\dot{\ell}_{n^{\epsilon}+2}>0$ and we can iterate the procedure to obtain (62). Hence, we can use Lemma 3.2. By (46), (47) and (63), we obtain the debonding evolution for $T \geq T_{2 n^{\epsilon}+1}^{\epsilon}$. In particular, $\dot{\ell}_{i}^{\epsilon}$ can be read as

$$
\epsilon \dot{\ell}_{i}^{\epsilon}=\frac{1-\left(X_{i-n^{\epsilon}-1}^{\epsilon}\right)^{2}}{1+\left(X_{i-n^{\epsilon}-1}^{\epsilon}\right)^{2}}
$$

and we have the results of convergence

$$
\lim _{i \rightarrow \infty} \dot{\ell}_{i}^{\epsilon}=\dot{\ell}_{\infty}^{\epsilon}:=\frac{1}{\sqrt{2 \gamma_{2}+\epsilon^{2}}}, \quad \lim _{i \rightarrow \infty} \omega_{i}^{\epsilon}=-\sqrt{2 \gamma_{2}+\epsilon^{2}}
$$

setting that the speed of debonding and the rotation of the film tend to those corresponding to the propagation in a homogeneous medium with toughness $\gamma_{2}$. The speed of debonding $\dot{\ell}_{i}^{\epsilon}$ oscillates around that limit value and tends exponentially to it (with $i$ ). That phenomenon of oscillation can be interpreted as a boundary layer effect generated by the phase of propagation into the first zone and the phase of arrest at the interface which modify the initial conditions corresponding to the problem of propagation into a homogeneous medium.

\subsubsection{Convergence to the quasi-static solution when $\epsilon \rightarrow 0$}

When the speed of loading goes down to 0 we obtain the following expected convergence result

Proposition 3.3 The dynamic solution $T \mapsto \ell^{\epsilon}(T)$ converges to the quasistatic solution $T \mapsto \ell(T)$ given by (29), uniformly on any compact, see Figure 7. Moreover, the potential and surface energies converge also uniformly on any compact to their quasi-static homologue given by (30)-(31), the kinetic energy converges to 0 .

Proof 
Step 1: Convergence of the first two phases.

We deduce from (55) and (56) that $\lim _{\epsilon \rightarrow 0} \dot{\ell}_{0}^{\epsilon}=1 / \sqrt{2 \gamma_{1}}$ and $\lim _{\epsilon \rightarrow 0} T_{1}^{\epsilon}=\sqrt{2 \gamma_{1}}$. Furthermore, since (59) gives $\lim _{\epsilon \rightarrow 0} 2 \epsilon n^{\epsilon}=\sqrt{2 \gamma_{2}}-\sqrt{2 \gamma_{1}}$, we also deduce from (60) that $\lim _{\epsilon \rightarrow 0} T_{2 n^{\epsilon}+1}^{\epsilon}=\sqrt{2 \gamma_{2}}$.

Step 2: Convergence of the third phase.

It is the most difficult part of the proof, because of the oscillations. We know from Lemma 3.2 that $\dot{\ell}_{i}^{\epsilon}$ oscillates around the limit speed $\left(2 \gamma_{2}+\epsilon^{2}\right)^{-1 / 2}$ and converges to it when $i \rightarrow \infty$. Let us define the mean value of the slope of the debonding front during one oscillation, i.e.

$$
p_{i}^{\epsilon}:=\frac{\ell_{i+2}^{\epsilon}-\ell_{i}^{\epsilon}}{T_{2 i+3}^{\epsilon}-T_{2 i-1}^{\epsilon}}, \quad i \geq 1 .
$$

Using (47), (62) and (65), we obtain after a tedious calculation

$$
\dot{\ell}_{\infty}^{\epsilon}-p_{i}^{\epsilon}=\frac{2 \dot{\ell}_{\infty}^{\epsilon}\left(X_{i-n^{\epsilon}-1}^{\epsilon}+\eta_{\epsilon}-\sqrt{1+\eta_{\epsilon}^{2}}\right)^{2}}{\left(X_{i-n^{\epsilon}-1}^{\epsilon}+2 \eta_{\epsilon}\right)^{2}+\left(X_{i-n^{\epsilon}-1}^{\epsilon}\right)^{2}+2} .
$$

Since $\left|X_{i}^{\epsilon}+\eta_{\epsilon}-\sqrt{1+\eta_{\epsilon}^{2}}\right|$ decreases as $i$ increases, at given $\epsilon$, (that quantity tends to 0 when $i \rightarrow \infty$,) it is less than $\left|X_{0}^{\epsilon}+\eta_{\epsilon}-\sqrt{1+\eta_{\epsilon}^{2}}\right|$ which is of the order of $\epsilon$. Therefore, denoting generically by $C$ any positive constant independent of $\epsilon$ and $i$, we have

$$
0 \leq \dot{\ell}_{\infty}^{\epsilon}-p_{i}^{\epsilon} \leq C \epsilon^{2}
$$

Furthermore, since $0 \leq\left(2 \gamma_{2}\right)^{-1 / 2}-\dot{\ell}_{\infty}^{\epsilon} \leq C \epsilon^{2}$ and $\left|T_{2 n^{\epsilon}-1}^{\epsilon}-\sqrt{2 \gamma_{2}}\right| \leq C \epsilon$, we get $\left|\ell^{\epsilon}(T)-\ell(T)\right| \leq C \epsilon T$ for $T \geq \sqrt{2 \gamma_{2}}$.

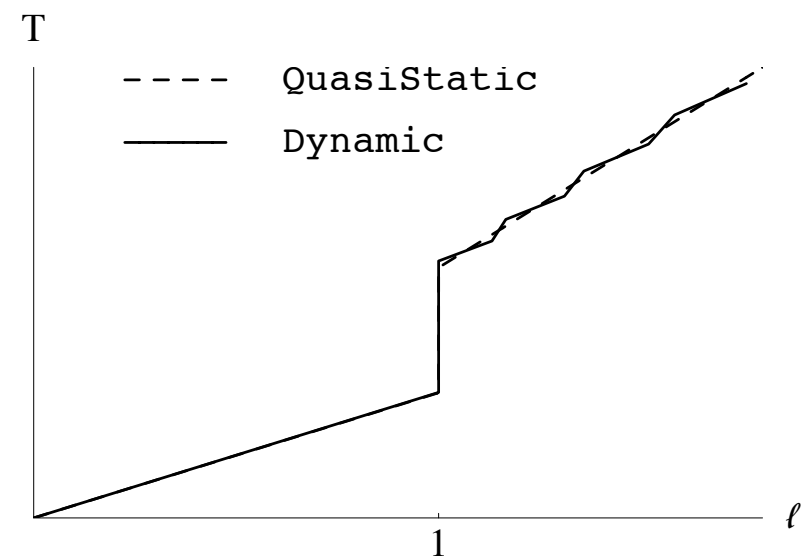

Fig. 7. Case a : Comparison of the dynamic solution when $\epsilon=0.075$ with the quasi-static solution for $\gamma_{1}=0.5, \gamma_{2}=2$.

Step 3: Convergence of the energies.

Since $\ell^{\epsilon}(T) \rightarrow \ell(T)$, we also have $\mathcal{S}^{\epsilon}(T) \rightarrow \mathcal{S}(T)$, uniformly on any compact. 
For the potential and the kinetic energies, we have for $1 \leq i \leq 2 n^{\epsilon}+1$ :

$$
\mathcal{P}^{\epsilon}\left(T_{i}^{\epsilon}\right)=\frac{N L}{2}\left(T_{i}^{\epsilon}\right)^{2}, \quad \mathcal{K}^{\epsilon}\left(T_{i}^{\epsilon}\right)= \begin{cases}\epsilon^{2} N L / 2, & \text { if } i \text { odd } \\ 0, & \text { if } i \text { even }\end{cases}
$$

with $T_{i}^{\epsilon}$ given by (61). Since $T_{1}^{\epsilon} \rightarrow \sqrt{2 \gamma_{1}}$, since $T_{2 n^{\epsilon}+1}^{\epsilon} \rightarrow \sqrt{2 \gamma_{2}}$, since the $T_{i}^{\epsilon}$ 's are equi-partitioned between $T_{1}^{\epsilon}$ and $T_{2 n^{\epsilon}+1}^{\epsilon}$ and since the energies are piecewise linear, we obtain the uniform convergence of $\mathcal{P}^{\epsilon}$ to $\mathcal{P}$ given by (30)-(31) and of $\mathcal{K}^{\epsilon}$ to 0 , in the interval $\left[0, \sqrt{2 \gamma_{2}}\right]$.

Since $\lim _{\epsilon \rightarrow 0} X_{j}^{\epsilon}=1$ for all $j \geq 0$ (with a uniform convergence with respect to $j$ ), we obtain from (46), (64) and (65) that $\lim _{\epsilon \rightarrow 0} \epsilon \dot{\ell}_{i}^{\epsilon}=0, \lim _{\epsilon \rightarrow 0} \epsilon v_{2 i-1}^{\epsilon}=$ $\lim _{\epsilon \rightarrow 0} \epsilon v_{2 i}^{\epsilon}=0$ and $\lim _{\epsilon \rightarrow 0} \omega_{2 i-1}^{\epsilon}=\lim _{\epsilon \rightarrow 0} \omega_{2 i}^{\epsilon}=\sqrt{2 \gamma_{2}}$, for all $i \geq n^{\epsilon}+1$ (with a uniform convergence with respect to $i$ ). Inserting that into (48) and (49), we obtain the uniform convergence of $\mathcal{P}^{\epsilon}$ to $\mathcal{P}$ given by (30)-(31) and of $\mathcal{K}^{\epsilon}$ to 0 for $T \geq \sqrt{2 \gamma_{2}}$.

\subsection{Case b}

\subsubsection{The dynamic solution}

The speed of loading $\epsilon$ is assumed to be small enough to remove some particular cases. We can divide the debonding evolution into four phases, see Figure 8.

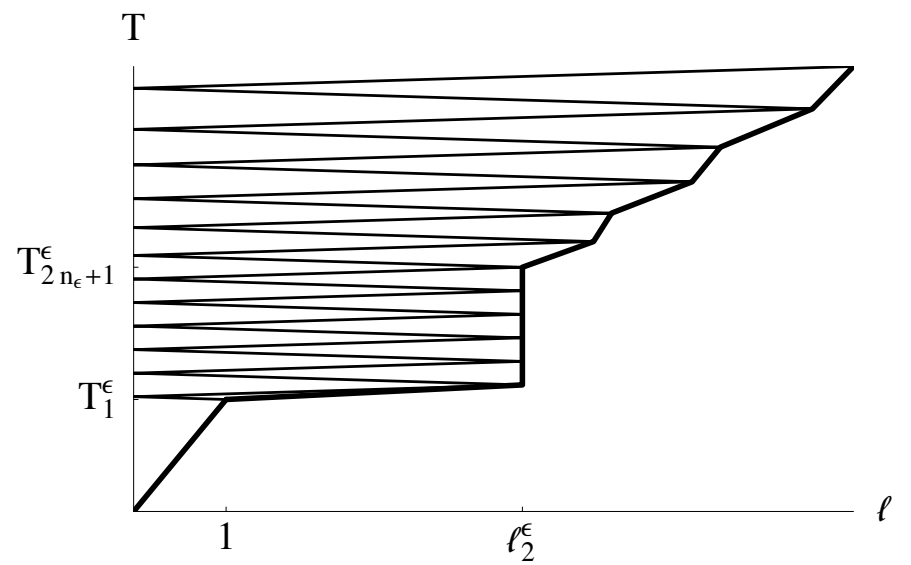

Fig. 8. Case $\mathbf{b}$ : Dynamic solution with the four phases of the debonding propagation for $\gamma_{1}=0.5, \gamma_{2}=2$. and $\epsilon=0.05$ 
3.3.1.1 Slow debonding in the zone with the lower toughness. In the sector $\mathcal{Q}_{1}^{\epsilon}$, (45) gives

$$
\dot{\ell}_{0}^{\epsilon}=\frac{1}{\sqrt{2 \gamma_{2}+\epsilon^{2}}}, \quad \omega_{1}^{\epsilon}=-\sqrt{2 \gamma_{2}+\epsilon^{2}}, \quad v_{1}^{\epsilon}=1, \quad T_{1}^{\epsilon}=\sqrt{2 \gamma_{2}+\epsilon^{2}} .
$$

For $T>T_{1}^{\epsilon}$, the debonding evolution is governed by Proposition 3.1.

\subsubsection{Rapid debonding in the zone with the higher toughness.}

There is major difference between the two cases when the front of debonding reaches the point $x=1$ where the toughness changes. In Case a since the toughness increases, the debonding stops. On the contrary, in Case b since the toughness decreases, the debonding accelerates. Specifically, we deduce from (46) that

$$
\epsilon \dot{\ell}_{1}^{\epsilon}=\frac{\left(\sqrt{2 \gamma_{2}+\epsilon^{2}}+\epsilon\right)^{2}-2 \gamma_{1}}{\left(\sqrt{2 \gamma_{2}+\epsilon^{2}}+\epsilon\right)^{2}+2 \gamma_{1}} .
$$

Hence, $\epsilon \dot{\ell}_{1}^{\epsilon}$ is less than 1 but of the order of 1 , i.e. the debonding propagates at a speed of the same order that the wave speed which remains an unreachable limit speed. The first backward wave reaches $x=0$ at $T_{2}^{\epsilon}=T_{1}^{\epsilon}+\epsilon$. Then, it is reflected and the generated forward shock wave intersects the front of debonding at $\left(\ell_{2}^{\epsilon}, T_{3}^{\epsilon}\right)$ given by

$$
\ell_{2}^{\epsilon}=\frac{1+\epsilon \dot{\ell}_{1}^{\epsilon}}{1-\epsilon \dot{\ell}_{1}^{\epsilon}}, \quad T_{3}^{\epsilon}=T_{1}^{\epsilon}+\frac{2 \epsilon}{1-\epsilon \dot{\ell}_{1}^{\epsilon}} .
$$

At $T_{1}^{\epsilon}$ and $T_{3}^{\epsilon}$, the kinetic energy is of the order of $\epsilon^{2}$ while it is of the order of 1 at $T_{2}^{\epsilon}$. Specifically, we have

$$
\begin{gathered}
\mathcal{K}^{\epsilon}\left(T_{1}^{\epsilon}\right)=\epsilon^{2} \frac{N L}{2}, \quad \mathcal{K}^{\epsilon}\left(T_{3}^{\epsilon}\right)=\epsilon^{2} \frac{1+\epsilon \dot{\ell}_{1}^{\epsilon}}{1-\epsilon \dot{\ell}_{1}^{\epsilon}} \frac{N L}{2} \\
\mathcal{K}^{\epsilon}\left(T_{2}^{\epsilon}\right)=\frac{\left(\epsilon \dot{\ell}_{1}^{\epsilon}\right)^{2}\left(\sqrt{2 \gamma_{2}+\epsilon^{2}}+\epsilon\right)^{2}}{1+\epsilon \dot{\ell}_{1}^{\epsilon}} \frac{N L}{2} .
\end{gathered}
$$

3.3.1.3 Arrest at the point $x=\ell_{2}^{\epsilon}$. At $T=T_{3}^{\epsilon}$, the debonding stops. Indeed, we have

$$
\left|\omega_{3}^{\epsilon}\right|=\frac{2 \gamma_{1}}{\sqrt{2 \gamma_{2}+\epsilon^{2}}+\epsilon}+\epsilon .
$$

Therefore, for $\epsilon$ small enough, since $\left|\omega_{3}^{\epsilon}\right|+\epsilon<\sqrt{2 \gamma_{1}}$, we deduce from (46) that $\dot{\ell}_{2}^{\epsilon}=0$. Furthermore, by virtue of (46) and (47) we get

$v_{4}^{\epsilon}=0, \quad \omega_{4}^{\epsilon}=-\frac{2 \gamma_{1}}{\sqrt{2 \gamma_{2}+\epsilon^{2}}+\epsilon}-2 \epsilon, \quad v_{5}^{\epsilon}=1, \quad \omega_{5}^{\epsilon}=-\frac{2 \gamma_{1}}{\sqrt{2 \gamma_{2}+\epsilon^{2}}+\epsilon}-3 \epsilon$. 
With the same procedure as in 3.2.1.2, using (46), we can show that

$$
\dot{\ell}_{i}^{\epsilon}=0, \quad v_{2 i}^{\epsilon}=0, \quad v_{2 i+1}^{\epsilon}=1, \quad 2 \leq i \leq n^{\epsilon}
$$

and

$$
\omega_{i}^{\epsilon}=-\frac{2 \gamma_{1}}{\sqrt{2 \gamma_{2}+\epsilon^{2}}+\epsilon}-(i-2) \epsilon, \quad 4 \leq i \leq 2 n^{\epsilon}+1
$$

with

$$
n^{\epsilon}=\left\langle\frac{1}{2 \epsilon}\left(\sqrt{2 \gamma_{1}}-\frac{2 \gamma_{1}}{\sqrt{2 \gamma_{2}+\epsilon^{2}}+\epsilon}+2 \epsilon\right)\right\rangle .
$$

In terms of $T$, the debonding does not evolve in the interval $\left(T_{3}^{\epsilon}, T_{2 n^{\epsilon}+1}^{\epsilon}\right)$ with

$$
T_{2 n^{\epsilon}+1}^{\epsilon}=T_{3}^{\epsilon}+2 \epsilon n^{\epsilon} \ell_{2}^{\epsilon}
$$

3.3.1.4 Slow debonding in the zone with the higher toughness. At $T=T_{2 n^{\epsilon}+1}^{\epsilon}$ the debonding restarts. Indeed, for $j \geq 0$ let us put

$$
X_{j}^{\epsilon}=\frac{\sqrt{2 \gamma_{1}}}{\left|\omega_{2 n^{\epsilon}+1+2 j}^{\epsilon}\right|+\epsilon}, \quad \eta_{\epsilon}=\frac{\epsilon}{\sqrt{2 \gamma_{1}}} .
$$

By definition of $n^{\epsilon}$, we have $\sqrt{2 \gamma_{1}}<\left|\omega_{2 n^{\epsilon}+1}^{\epsilon}\right|+\epsilon \leq \sqrt{2 \gamma_{1}}+2 \epsilon$ and hence, for $\epsilon$ small enough, $0<1-2 \eta_{\epsilon}<\left(1+2 \eta_{\epsilon}\right)^{-1} \leq X_{0}^{\epsilon}<1$. Furthermore, since $\dot{\ell}_{n^{\epsilon}+1}^{\epsilon}>0$, we get from (46) and (47), $1 / X_{1}^{\epsilon}=X_{0}^{\epsilon}+2 \eta_{\epsilon}$. Since $0<X_{1}^{\epsilon}<1$, we have $\dot{\ell}_{n^{\epsilon}+2}^{\epsilon}>0$ and by induction we obtain $1 / X_{j+1}^{\epsilon}=X_{j}^{\epsilon}+2 \eta_{\epsilon}$. We can use Lemma 3.2. Owing to (46), (47) and (63), we obtain the debonding evolution for $T \geq T_{2 n^{\epsilon}+1}^{\epsilon}$. In particular, for $i \geq n^{\epsilon}+1, \dot{\ell}_{i}^{\epsilon}$ can be read as

$$
\epsilon \dot{\ell}_{i}^{\epsilon}=\frac{1-\left(X_{i-n^{\epsilon}-1}^{\epsilon}\right)^{2}}{1+\left(X_{i-n^{\epsilon}-1}^{\epsilon}\right)^{2}}
$$

and we get the convergence result

$$
\lim _{i \rightarrow \infty} \dot{\ell}_{i}^{\epsilon}=\dot{\ell}_{\infty}^{\epsilon} \equiv \frac{1}{\sqrt{2 \gamma_{1}+\epsilon^{2}}}
$$

The speed of debonding $\dot{\ell}_{i}^{\epsilon}$ oscillates around this limit value and tends exponentially to it (with respect to $i$ ).

\subsubsection{Convergence to the quasi-static solution when $\epsilon \rightarrow 0$}

We pass to the limit when $\epsilon$ goes down to 0 and compare the limits of the dynamic solution to that of the conjectured quasi-static response given by (34). 


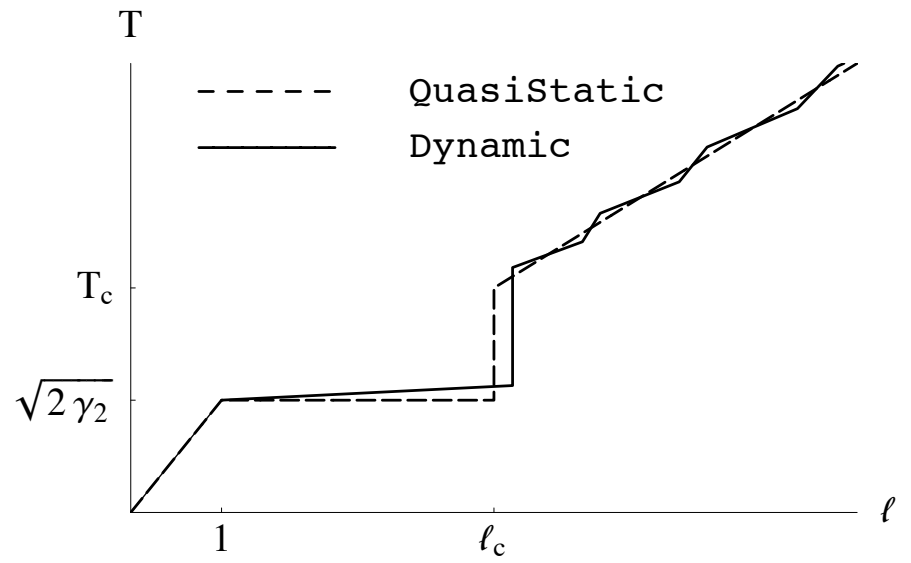

Fig. 9. Case b : Comparison of the dynamic solution when $\epsilon=0.05$ with the quasi-static solution for $\gamma_{1}=0.5, \gamma_{2}=2$.

3.3.2.1 Convergence of the first phase. We deduce from (68) that $\lim _{\epsilon \rightarrow 0} \dot{\ell}_{0}^{\epsilon}=1 / \sqrt{2 \gamma_{2}}$ and $\lim _{\epsilon \rightarrow 0} T_{1}^{\epsilon}=\sqrt{2 \gamma_{2}}$. Hence, the convergence to the corresponding quasi-static first phase. Let us note that the kinetic energy converges to 0 like $\epsilon^{2}$.

\subsubsection{Convergence of the second phase to the predicted jump.} Let us remark first that

$$
\lim _{\epsilon \rightarrow 0} \epsilon \dot{\ell}_{1}^{\epsilon}=\frac{\gamma_{2}-\gamma_{1}}{\gamma_{2}+\gamma_{1}}
$$

then that

$$
\lim _{\epsilon \rightarrow 0} T_{1}^{\epsilon}=\lim _{\epsilon \rightarrow 0} T_{3}^{\epsilon}=\sqrt{2 \gamma_{2}}, \quad \lim _{\epsilon \rightarrow 0} \ell_{2}^{\epsilon}=\frac{\gamma_{2}}{\gamma_{1}} .
$$

Therefore, the second phase tends to the jump of the debonding length from $\ell=1$ to $\ell=\ell_{c}$ at $T=\sqrt{2 \gamma_{2}}$ as in (34). This convergence result can be interpreted in terms of the energies. Thus, at $T_{1}^{\epsilon}, T_{2}^{\epsilon}$ and $T_{3}^{\epsilon}$, the kinetic energy takes the values

$$
\mathcal{K}_{\epsilon}\left(T_{1}^{\epsilon}\right)=\epsilon^{2} \frac{N L}{2}, \quad \mathcal{K}_{\epsilon}\left(T_{3}^{\epsilon}\right)=\epsilon^{2} \frac{1+\epsilon \dot{\ell}_{1}^{\epsilon}}{1-\epsilon \dot{\ell}_{1}^{\epsilon}} \frac{N L}{2}
$$

and

Therefore, at the limit

$$
\mathcal{K}_{\epsilon}\left(T_{2}^{\epsilon}\right)=\frac{\left(\epsilon \dot{\ell}_{1}^{\epsilon}\right)^{2}\left(\sqrt{2 \gamma_{2}+\epsilon^{2}}+\epsilon\right)^{2}}{1+\epsilon \dot{\ell}_{1}^{\epsilon}} \frac{N L}{2} .
$$

$$
\lim _{\epsilon \rightarrow 0} \mathcal{K}_{\epsilon}\left(T_{1}^{\epsilon}\right)=\lim _{\epsilon \rightarrow 0} \mathcal{K}_{\epsilon}\left(T_{3}^{\epsilon}\right)=0, \quad \lim _{\epsilon \rightarrow 0} \mathcal{K}_{\epsilon}\left(T_{2}^{\epsilon}\right)=\frac{\left(\gamma_{2}-\gamma_{1}\right)^{2}}{\gamma_{2}+\gamma_{1}} \frac{N L}{2} .
$$

For the surface energy, we have

$$
\mathcal{S}_{\epsilon}\left(T_{1}^{\epsilon}\right)=\gamma_{2} N L, \quad \mathcal{S}_{\epsilon}\left(T_{3}^{\epsilon}\right)=\left(\gamma_{2}+\frac{2 \epsilon \dot{\ell}_{1}^{\epsilon}}{1-\epsilon \dot{\ell}_{1}^{\epsilon}} \gamma_{1}\right) N L
$$


and at the limit

$$
\lim _{\epsilon \rightarrow 0} \mathcal{S}_{\epsilon}\left(T_{3}^{\epsilon}\right)=\left(2 \gamma_{2}-\gamma_{1}\right) N L .
$$

The potential energy takes the following values

$$
\begin{aligned}
& \mathcal{P}_{\epsilon}\left(T_{1}^{\epsilon}\right)=\left(2 \gamma_{2}+\epsilon^{2}\right) \frac{N L}{2} \\
& \mathcal{P}_{\epsilon}\left(T_{3}^{\epsilon}\right)=\left(\frac{1-\epsilon \dot{\ell}_{1}^{\epsilon}}{1+\epsilon \dot{\ell}_{1}^{\epsilon}}\left(\sqrt{2 \gamma_{2}+\epsilon^{2}}+\epsilon\right)+\epsilon\right)^{2} \frac{1+\epsilon \dot{\ell}_{1}^{\epsilon}}{1-\epsilon \dot{\ell}_{1}^{\epsilon}} \frac{N L}{2}
\end{aligned}
$$

and at the limit

$$
\lim _{\epsilon \rightarrow 0} \mathcal{P}_{\epsilon}\left(T_{1}^{\epsilon}\right)=\gamma_{2} N L, \quad \lim _{\epsilon \rightarrow 0} \mathcal{P}_{\epsilon}\left(T_{3}^{\epsilon}\right)=\gamma_{1} N L .
$$

While comparing to (37) and (38), we see that the limits of the initial values

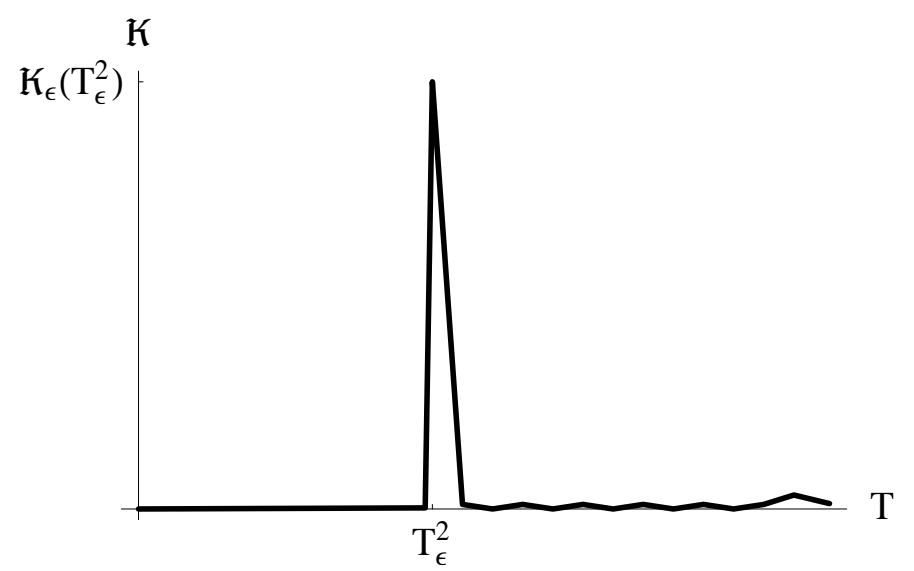

Fig. 10. Evolution of the kinetic energy with $T$ when $\gamma_{1}=0.5, \gamma_{2}=2$. and $\epsilon=0.05$.

and of the final values are those of the quasi-static response, because the kinetic energy is then negligible. However, the kinetic energy is not always negligible during this phase because it takes a finite limit at $T_{2}^{\epsilon}$. Specifically, let us examine the evolution of the kinetic energy. It is negligible during the first phase. Then, it grows rapidly during the propagation of the first backward shock wave to become maximal when that wave is reflected at $\mathrm{x}=0$. But the kinetic energy decreases rapidly during the propagation of the first forward shock wave to become negligible again when that wave intersects the front of debonding, see Figure 10. During this come back of the wave, all the kinetic energy is transformed into surface energy. Finally, the jump of the debonding satisfies the principle of conservation of the quasi-static energy.

3.3.2.3 Convergence of the phase of arrest. Passing to the limit in (76) and (77) we get

$$
\lim _{\epsilon \rightarrow 0} T_{2 n^{\epsilon}+1}^{\epsilon}=\frac{2 \gamma_{2}}{\sqrt{2 \gamma_{1}}}=T_{c}
$$


Hence, the phase arrest converges to that of the quasi-static solution. Since $v_{i}^{\epsilon}$ oscillates between 0 and 1 during that phase, the kinetic energy converges to 0 as $\epsilon^{2}$. We easily deduce that the other energies converge to their quasi-static homologue.

3.3.2.4 Convergence of the last phase. The proof that the dynamic last phase (where the debonding restarts and propagates with a oscillating speed) converges to the quasi-static last phase is quite similar to that given in Case a, see Step 2 of Proposition 3.3. During this phase the kinetic energy remains of the order of $\epsilon^{2}$ and all the energies converge to their quasi-static homologue. We can summarize the convergence result obtained in Case $\mathbf{b}$ by

Proposition 3.4 When the speed of loading $\epsilon$ goes down to 0, the dynamic solution of Case $\mathbf{b}$ converges to the extended quasi-static solution (34). Thus the evolution is given by the quasi-static Griffith's law during the first, third and fourth phases where the debonding evolution is continuous, but the second phase leads to a jump of the debonding which satisfies the principle of conservation of the total quasi-static energy. The role of the kinetic energy is transitory. It takes finite values only during the first back and forth of the shock waves, being negligible before and after the jump.

\section{Conclusion}

We can learn two major lessons from this model problem of dynamic fracture:

(1) Contrary to what is generally claimed, we can treat the phase of rapid propagation by considering only quasi-static quantities, because the kinetic energy plays only a transitory role.

(2) The right criterion giving the value of the crack jump is not Griffith's criterion formulated in terms of the energy release rate but the conservation of the total energy.

Of course, it remains to generalize those results to two- or three-dimensional heterogeneous bodies. In particular, by considering layered composite materials we could obtain their effective toughness.

\section{Acknowledgements}

We thank the referee who has carefully read the paper and who has verified each formula and each proof. His remarks have allowed us to improve the 
quality of the paper.

\section{A Definition of the dynamic potential energy release rate}

Let us consider a two-dimensional homogeneous elastic medium in which a crack propagates in the direction 1 . In dynamics, the potential energy release rate $G$ can be defined by the limit of an integral over a path which tends to the tip of the crack. Let $\Gamma_{r}$ be a such path - for instance a circle of radius $r$ and of center the tip of the crack - $G$ can be read as (cf [12])

$$
G=\lim _{r \rightarrow 0} \int_{\Gamma_{r}}\left(\left(\frac{\rho}{2} \dot{u}_{i} \dot{u}_{i}+W(\varepsilon(u))\right) n_{1}-\sigma_{i j} n_{j} \frac{\partial u_{i}}{\partial x_{1}}\right) d s,
$$

where $\rho$ is the mass density of the material, $\dot{u}$ the velocity vector field, $u$ the displacement vector field, $\varepsilon(u)$ the strain tensor field, $W$ the elastic potential, $\sigma$ the stress tensor field and $n$ the output unit normal to the path. The dynamic energy release rate differs from its static homologue by the first term corresponding to the kinetic energy.

This relationship of $G$ can be used for the peeling test as follows. Let $\ell$ be the position of the debonding tip. The path $\Gamma_{r}$ corresponds to the points $\{\ell-r, \ell+r\}$, the normal to the path at $\ell \pm r$ is equal to \pm 1 , all the indices take the value 1 , the displacement $u$ corresponds to the opening $w$ and the strain reads as $\varepsilon=w^{\prime}$. Assuming that the elastic potential is quadratic, $W\left(w^{\prime}\right)=$ $N w^{\prime 2} / 2$, the stress becomes $\sigma=N w^{\prime}$ and the dynamic energy release rate reads as

$$
G=\frac{\rho}{2}\left(\dot{w}(\ell+)^{2}-\dot{w}(\ell-)^{2}\right)-\frac{N}{2}\left(w^{\prime}(\ell+)^{2}-w^{\prime}(\ell-)^{2}\right),
$$

where the dot denotes the time derivative and the prime the spatial derivative. Furthermore, if we assume that the debonding grows in the direction 1 and if the front of debonding is oriented in the space-time so that the + side corresponds to the points after the passage of the tip debonding, we get

$$
G=\frac{N}{2} \llbracket w^{\prime 2} \rrbracket-\frac{\rho}{2} \llbracket \dot{w}^{2} \rrbracket,
$$

remaining that the double brackets denote the jump.

\section{References}

[1] J. Berry, Some kinetic considerations of the Griffith criterion for fracture I: Equations of motion at constant force, J. Mech. Phys. Solids 8 (3) (1960) 
$194-206$.

[2] J. Berry, Some kinetic considerations of the Griffith criterion for fracture - II: Equations of motion at constant deformation, J. Mech. Phys. Solids 8 (3) (1960) 207-216.

[3] A. Mielke, Evolution of rate-independent systems, in: Evolutionary equations, Vol. II of Handb. Differ. Equ., Elsevier/North-Holland, Amsterdam, 2005, pp. 461-559.

[4] G. A. Francfort, A. Mielke, Existence results for a class of rate-independent material models with nonconvex elastic energies, J. Reine Angew. Math. 595 (2006) 55-91.

[5] B. Bourdin, G. A. Francfort, J.-J. Marigo, The variational approach to fracture, J. Elasticity to appear.

[6] J. W. Obreimoff, The splitting strength of mica, Proceedings of the Royal Society (London) A127 (1930) 290-297.

[7] B. Lawn, Fracture of Brittle Solids - Second Edition, Cambridge Solid State Science Series, Cambridge University press, Cambridge, 1993.

[8] A. Jaubert, J.-J. Marigo, Justification of Paris-type fatigue laws from cohesive forces model via a variational approach, Continuum Mechanics and Thermodynamics V18 (1) (2006) 23-45.

[9] A. Griffith, The phenomena of rupture and flow in solids, Philos. T. Roy. Soc. A CCXXI-A (1920) 163-198.

[10] J. D. Achenbach, Wave Propagation in Elastic Solids, North-Holland, Amsterdam, 1973.

[11] C. Truesdell, R. Toupin, The Classical Field Theories, in: S. Flügge (Ed.), Handbuch der Physik, Vol. III/1, Springer-Verlag, Berlin, 1960.

[12] L. B. Freund, Dynamic Fracture Mechanics, Cambridge Monographs on Mechanics and Applied Mathematics, Cambridge University Press, 1998.

[13] H. D. Bui, Mécanique de la Rupture Fragile, Masson, Paris, 1978.

[14] L. Evans, R. Gariepy, Measure theory and fine properties of functions, CRC Press, Boca Raton, FL, 1992.

[15] G. A. Francfort, J.-J. Marigo, Revisiting brittle fracture as an energy minimization problem, J. Mech. Phys. Solids 46 (8) (1998) 1319-1342.

[16] Q. S. Nguyen, Stability and Nonlinear Solid Mechanics, Wiley \& Son, London, 2000 . 